\title{
Exponentially improved asymptotic solutions of ordinary differential equations. II Irregular singularities of rank one
}

\author{
By A. B. Olde DaAleuIS AND F. W. J. OlveR \\ ${ }^{1}$ CWI, P.O. Box 4079, 1009 AB Amsterdam, The Netherlands \\ ${ }^{2}$ Institute for Physical Science and Technology, \\ University of Maryland, College Park, \\ Maryland 20742, U.S.A.
}

Re-expansions are found for the optimal remainder terms in the well-known asymptotic series solutions of homogeneous linear differential equations of the second order in the neighbourhood of an irregular singularity of rank one. The re-expansions are in terms of generalized exponential integrals and have greater regions of validity than the original expansions, as well being considerably more accurate and providing a smooth interpretation of the Stokes phenomenon. They are also of strikingly simple form. In addition, explicit asymptotic expansions for the higher coefficients of the original asymptotic solutions are obtained.

\section{Introduction}

The general homogeneous linear differential equation of the second order is given by

$$
L w=0,
$$

where the operator $L$ is of the form

$$
L=\frac{\mathrm{d}^{2}}{\mathrm{~d} z^{2}}+f(z) \frac{\mathrm{d}}{\mathrm{d} z}+g(z) .
$$

If, as we shall suppose, the point at infinity is an irregular singularity of rank one, then the functions $f(z)$ and $g(z)$ can be expanded in power series

$$
f(z)=\sum_{s=0}^{\infty} \frac{f_{s}}{z^{s}}, \quad g(z)=\sum_{s=0}^{\infty} \frac{g_{s}}{z^{s}}
$$

that converge in an unbounded open annulus $\boldsymbol{A}$ centred at the origin. Not all of the coefficients $f_{0}, g_{0}$ and $g_{1}$ vanish, otherwise infinity would be a regular singularity.

The asymptotic theory of solutions of (1.1) in these circumstances is well known and will be found, for example, in Olver (1974, ch. 7, §§1-2). Following the notations used in this reference, we shall assume, without loss of generality, that the

Proc. R. Soc. Lond. A (1994) 445, 39-56

(c) 1994 The Royal Society 
roots $\lambda_{1}$ and $\lambda_{2}$ of the characteristic equation

$$
\lambda^{2}+f_{0} \lambda+g_{0}=0
$$

are distinct. $\nmid$ Then equation (1.1) has unique solutions $w_{1}(z)$ and $w_{2}(z)$ such that

$$
w_{1}(z) \sim \mathrm{e}^{\lambda_{1} z} z^{\mu_{1}} \sum_{s=0}^{\infty} \frac{a_{s, 1}}{z^{s}},
$$

as $z \rightarrow \infty$ in the sector $\left|\operatorname{ph}\left\{\left(\lambda_{2}-\lambda_{1}\right) z\right\}\right| \leqslant \frac{3}{2} \pi-\delta$, and

$$
w_{2}(z) \sim \mathrm{e}^{\lambda_{2} z} z^{\mu_{2}} \sum_{s=0}^{\infty} \frac{a_{s, 2}}{z^{s}},
$$

as $z \rightarrow \infty$ in the sector $\left|\operatorname{ph}\left\{\left(\lambda_{1}-\lambda_{2}\right) z\right\}\right| \leqslant \frac{3}{2} \pi-\delta$. Here (and elsewhere in this paper) $\delta$ denotes an arbitrary small positive constant, and the exponents $\mu_{1}, \mu_{2}$ and the coefficients $a_{s, 1}, a_{s, 2}$ may be found by formal substitution in (1.1) (see $\S 2$ below). Any branches may be taken for $z^{\mu_{1}}$ and $z^{\mu_{2}}$, provided that they are continuous and used consistently throughout.

In the special case in which $L$ is the confluent hypergeometric differential operator, it is known (Olver 1991b, 1993; Paris 1992b) that the remainder terms associated with the expansions (1.5) and (1.6) can be re-expanded in such a way that these expansions are exponentially improved in the sense defined in Olver $(1991 a, b)$; furthermore, the sectors of validity are increased. The purpose of this paper is to develop a similar theory for the more general case. Earlier work on this problem includes the formal research of Berry (1990) and the rigorous analysis of Paris (1992a). The latter applies to certain differential equations that can be solved exactly in terms of Mellin-Barnes integrals. The investigation whose aims are closest to those of this paper is that of McLeod (1992); however, McLeod's results are neither so general nor so powerful as the ones we shall develop.

As in the earlier references, the re-expansions of the remainder terms will be expressed in terms of generalized exponential integrals (or incomplete Gamma functions), defined by

$$
F_{p}(z)=\frac{\mathrm{e}^{-z}}{2 \pi} \int_{0}^{\infty} \frac{\mathrm{e}^{-z t} t^{p-1}}{1+t} \mathrm{~d} t,
$$

when $\Re p>0$ and $|\mathrm{ph} z|<\frac{1}{2} \pi$, and by analytic continuation elsewhere. Relevant properties of these functions will be found in Olver $(1993, \S 2)$.

The original intention was to use a direct differential-equation approach as in the special case treated in Olver (1993). However, significant difficulties appear in the analysis that are absent from the proofs in that reference. Thus it is necessary, at first, to match the optimal remainder terms by a double series of $F$ functions, rather than a single series; furthermore, the coefficients in the double series are unavailable in simple explicit form. To prove that the double series may be reduced to a single series, with explicit coefficients, elaborate indirect arguments based on limiting forms of the double series have to be used.

The approach in this paper is quite different. By using the connection formu-

$\dagger$ The case $\lambda_{1}=\lambda_{2}$ can always be handled by application of a preliminary transformation due to Fabry (Olver 1974, ch. 7, $\$ 1.3$ ).

Proc. R. Soc. Lond. A (1994) 
lae for the solutions and contour integration we construct Stieltjes-type transforms for the remainder terms, from which the desired re-expansions can be found directly. N Not only is the analysis considerably simpler; it will lend itself more readily to further developments and extensions. These include higher re-expansions of remainder terms (hyperasymptotics), and differential equations of other orders or with other types of singularity.

We should also add that if the theorems of this paper are applied to the confluent hypergeometric equation, then the results agree with those of Olver (1993).

\section{Main results}

If we replace the independent variable $z$ by $z /\left(\lambda_{2}-\lambda_{1}\right)$, then the essential form (1.2) of the differential operator $L$ is unchanged, but the new characteristic values satisfy

$$
\lambda_{2}-\lambda_{1}=1
$$

The effect of this transformation is to simplify considerably the notation in subsequent analyses; in consequence, throughout the rest of this paper we shall assume that the condition (2.1) is satisfied.

The branches of the asymptotic solutions are chosen to be

$$
w_{1}(z) \sim \mathrm{e}^{\lambda_{1} z} z^{\mu_{1}} \sum_{s=0}^{\infty} \frac{a_{s, 1}}{z^{s}}, \quad|\mathrm{ph} z| \leqslant \frac{3}{2} \pi-\delta
$$

and

$$
w_{2}(z) \sim \mathrm{e}^{\lambda_{2} z} z^{\mu_{2}} \sum_{s=0}^{\infty} \frac{a_{s, 2}}{z^{s}}, \quad-\frac{1}{2} \pi+\delta \leqslant \mathrm{ph} z \leqslant \frac{5}{2} \pi-\delta
$$

compare (1.5) and (1.6). The characteristic values $\lambda_{1}, \lambda_{2}$ are the roots of the quadratic equation (1.4), and the exponents $\mu_{1}$ and $\mu_{2}$ are given by

$$
\mu_{1}=f_{1} \lambda_{1}+g_{1}, \quad \mu_{2}=-\left(f_{1} \lambda_{2}+g_{1}\right) .
$$

The coefficients are determined by $a_{0,1}=a_{0,2}=1$ and, when $s \geqslant 1$,

$$
\begin{aligned}
-s a_{s, 1}=\left(s-\mu_{1}\right)\left(s-1-\mu_{1}\right) a_{s \sim 1,1} & \\
& +\sum_{j=1}^{s}\left\{\lambda_{1} f_{j+1}+g_{j+1}-\left(s-j-\mu_{1}\right) f_{j}\right\} a_{s-j, 1} \\
s a_{s, 2}=\left(s-\mu_{2}\right)\left(s-1-\mu_{2}\right) & a_{s-1,2} \\
& +\sum_{j=1}^{s}\left\{\lambda_{2} f_{j+1}+g_{j+1}-\left(s-j-\mu_{2}\right) f_{j}\right\} a_{s-j, 2} .
\end{aligned}
$$

A direct consequence of (1.3) is that $w_{1}\left(z \mathrm{e}^{-2 \pi i}\right)$ and $w_{2}\left(z \mathrm{e}^{2 \pi i}\right)$ are also solutions of (1.1). Note that $w_{1}(z)$ and $\mathrm{e}^{2 \pi i \mu_{1}} w_{1}\left(z \mathrm{e}^{-2 \pi i}\right)$ are dominant solutions in the sector

$\ddagger$ These transforms can be regarded as 'resurgence relations' in the terminology of Berry (1991) and Berry \& Howls (1991). Perhaps it should be noted that Stieltjes transforms were used by Olver (1990) and Boyd (1990), respectively, in deriving exponentially improved asymptotic expansions for the confuent hypergeometric function and a modified Bessel function via integral-representation approaches.

Proc. R. Soc. Lond. A (1994) 
$\frac{1}{2} \pi+\delta \leqslant \operatorname{ph} z \leqslant \frac{3}{2} \pi-\delta$ and have exactly the same asymptotic expansion there. Similarly for $w_{2}(z)$ and $\mathrm{e}^{-2 \pi i \mu_{2}} w_{2}\left(z \mathrm{e}^{2 \pi i}\right)$ in the sector $|\mathrm{ph} z| \leqslant \frac{1}{2} \pi-\delta$. Thus there are constants $C_{1}$ and $C_{2}$ such that

$$
\begin{aligned}
& w_{1}(z)=\mathrm{e}^{2 \pi i \mu_{1}} w_{1}\left(z \mathrm{e}^{-2 \pi i}\right)+C_{1} w_{2}(z), \\
& w_{2}(z)=\mathrm{e}^{-2 \pi i \mu_{2}} w_{2}\left(z \mathrm{e}^{2 \pi i}\right)+C_{2} w_{1}(z) .
\end{aligned}
$$

Formulae (2.7) and (2.8) are called the connection formulae, and we assume the constants $C_{1}$ and $C_{2}$ to be known.

The remainder of this paper is devoted to the proof of the following theorems.

Theorem 2.1. Let $m$ be an arbitrary fixed non-negative integer. Then as $s \rightarrow \infty$

$$
\begin{aligned}
a_{s, 1}= & (-)^{s} \frac{e^{\left(\mu_{2}-\mu_{1}\right) \pi i}}{2 \pi i} \\
& \times\left\{C_{1} \sum_{j=0}^{m-1}(-)^{j} a_{j, 2} \Gamma\left(s+\mu_{2}-\mu_{1}-j\right)+\Gamma\left(s+\mu_{2}-\mu_{1}-m\right) \mathcal{O}(1)\right\}, \\
a_{s, 2}= & -\frac{1}{2 \pi i}\left\{C_{2} \sum_{j=0}^{m-1} a_{j, 1} \Gamma\left(s+\mu_{1}-\mu_{2}-j\right)+\Gamma\left(s+\mu_{1}-\mu_{2}-m\right) \mathcal{O}(1)\right\} .(2 .
\end{aligned}
$$

Theorem 2.2. Define $R_{n}^{(1)}(z)$ and $R_{n}^{(2)}(z)$ by

$$
\begin{aligned}
& w_{1}(z)=\mathrm{e}^{\lambda_{1} z} z^{\mu_{1}} \sum_{s=0}^{n-1} \frac{a_{s, 1}}{z^{s}}+R_{n}^{(1)}(z), \\
& w_{2}(z)=\mathrm{e}^{\lambda_{2} z} z^{\mu_{2}} \sum_{s=0}^{n-1} \frac{a_{s, 2}}{z^{s}}+R_{n}^{(2)}(z),
\end{aligned}
$$

where

and $\alpha$ is bounded as $|z| \rightarrow \infty$. Then

$$
n=|z|+\alpha
$$

$$
\begin{aligned}
& R_{n}^{(1)}(z)=(-)^{n-1} i \mathrm{e}^{\left(\mu_{2}-\mu_{1}\right) \pi i} \mathrm{e}^{\lambda_{2} z} z^{\mu_{2}} \\
& \times\left\{C_{1} \sum_{s=0}^{m-1}(-)^{s} a_{s, 2} \frac{F_{n+\mu_{2}-\mu_{1}-s}(z)}{z^{s}}+R_{m, n}^{(1)}(z)\right\}, \\
& R_{n}^{(2)}(z)=(-)^{n} i \mathrm{e}^{\left(\mu_{2}-\mu_{1}\right) \pi i} \mathrm{e}^{\lambda_{1} z} z^{\mu_{1}} \\
& \times\left\{C_{2} \sum_{s=0}^{m-1}(-)^{s} a_{s, 1} \frac{F_{n+\mu_{1}-\mu_{2}-s}\left(z \mathrm{e}^{-\pi i}\right)}{z^{s}}+R_{m, n}^{(2)}(z)\right\}
\end{aligned}
$$

where $m$ is an arbitrary fixed non-negative integer, and for large $|z|$

$$
\begin{aligned}
& R_{m, n}^{(1)}(z)=\mathcal{O}\left(\mathrm{e}^{-|z|-z} z^{-m}\right), \quad|\mathrm{ph} z| \leqslant \pi, \\
& R_{m, n}^{(1)}(z)=\mathcal{O}\left(z^{-m}\right), \quad \pi \leqslant|\mathrm{ph} z| \leqslant \frac{5}{2} \pi-\delta, \\
& R_{m, n}^{(2)}(z)=\mathcal{O}\left(\mathrm{e}^{-|z|+z} z^{-m}\right), \quad 0 \leqslant \mathrm{ph} z \leqslant 2 \pi, \\
& R_{m, n}^{(2)}(z)=\mathcal{O}\left(z^{-m}\right), \quad-\frac{3}{2} \pi+\delta \leqslant \operatorname{ph} z \leqslant 0 \text { and } 2 \pi \leqslant \operatorname{ph} z \leqslant \frac{7}{2} \pi-\delta,(2.19)
\end{aligned}
$$

Proc. R. Soc. Land. A (1994) 
uniformly with respect to $\mathrm{ph} z$ in each case.

Furthermore if $C_{1}$ and $C_{2}$ are both non-zero, then the sectors of validity are maximal.

Remark (i). The results (2.9) and (2.10) are investigated in Olver (1994) using elementary analysis based on the recurrence relations (2.5) and (2.6).

Remark (ii). In consequence of (2.9) and (2.10), when $C_{1}$ and $C_{2}$ are non-zero the condition that $\alpha$ be bounded in equation (2.13) includes the situation in which the series in (2.11) and (2.12) are truncated at their optimal stages.

Remark (iii). At first sight it may seem remarkable that the coefficients $a_{s, 2}$ that appear in the re-expansion (2.14) of the optimal remainder term in the expansion (2.2) for $w_{1}(z)$ are the same as those in the expansion (2.3) for $w_{2}(z)$. One way to understand this phenomenon is as follows. If an expansion of the form (2.14) holds throughout the sector $|\mathrm{ph} z| \leqslant \frac{5}{2} \pi-\delta$, then from (2.17) and Olver (1993, eqn (2.11)), we see that in the outermost parts of this sector the contribution of (2.14) is dominant when compared with the finite sum in (2.11) and must therefore match the expansions for $w_{1}(z)$ in these outer regions obtained by use of connection formulae typified by (2.7). A similar observation applies to the coefficients $a_{s, 1}$ in (2.15).

Remark (iv). Corresponding results for other phase ranges can be derived easily from the stated results. For example, since $w_{2}\left(z \mathrm{e}^{2 \pi i}\right)$ is another solution of (1.1), on replacing $z$ by $z \mathrm{e}^{2 \pi i}$ throughout (2.12), (2.15), (2.18) and (2.19), we obtain a similar expansion for a solution in the phase range $-\frac{7}{2} \pi+\delta \leqslant \mathrm{ph} z \leqslant \frac{3}{2} \pi-\delta$.

Remark (v). If $C_{1}=0$, then equations (2.14), (2.16), (2.17) combine into

$$
R_{n}^{(1)}(z)=\mathcal{O}\left(\mathrm{e}^{-|x|+\lambda_{1} z} z^{\mu_{2}-m}\right), \quad|\mathrm{ph} z| \leqslant \pi
$$

and

$$
R_{n}^{(1)}(z)=\mathcal{O}\left(\mathrm{e}^{\lambda_{2} z} z^{\mu_{2}-m}\right), \quad \pi \leqslant|\mathrm{ph} z| \leqslant \frac{5}{2} \pi-\delta,
$$

for any integer $m$; compare (2.1). Actually, a stronger result applies in this case and it can be derived directly as follows.

From (2.7) we see that when $C_{1}=0$ we have

$$
w_{1}(z)=\mathrm{e}^{2 \pi i \mu_{1}} w_{1}\left(z \mathrm{e}^{-2 \pi i}\right) .
$$

Accordingly, the function $\mathrm{e}^{-\lambda_{1} z} z^{-\mu_{1}} w_{1}(z)$ is single-valued and analytic on $\boldsymbol{A}$. In consequence (Olver 1974, ch. $1, \S 7.5$ ) the expansion

$$
w_{1}(z)=\mathrm{e}^{\lambda_{1} z} z^{\mu_{1}} \sum_{s=0}^{\infty} \frac{a_{s, 1}}{z^{s}}
$$

converges on $\boldsymbol{A}$. Let $\rho$ be any constant that exceeds the radius of the boundary of $A$. Since $a_{s, 1} / \rho^{s} \rightarrow 0$ as $s \rightarrow \infty$, it follows that

$$
\left|a_{s, 1}\right| \leqslant H \rho^{s},
$$

where $H$ is assignable independently of $s$. Hence if $|z|>\rho$ we have

$$
\begin{aligned}
\left|R_{n}^{(1)}(z)\right|=\left|\mathrm{e}^{\lambda_{1} z} z^{\mu_{1}} \sum_{s=n}^{\infty} \frac{a_{s, 1}}{z^{s}}\right| & \leqslant H\left|\mathrm{e}^{\lambda_{1} z} z^{\mu_{1}}\right|\left(\frac{\rho}{|z|}\right)^{n} \frac{|z|}{|z|-\rho} \\
& =\left(\frac{\rho}{|z|}\right)^{|z|} \mathrm{e}^{\lambda_{1} z} z^{\mu_{1}-\alpha} \mathcal{O}(1) ;
\end{aligned}
$$

Proc. R. Soc. Lond. A (1994) 
compare (2.13). Furthermore, this estimate applies without restriction on $\mathrm{ph} z$.

Similarly, (2.18) and (2.19) may be strengthened when $C_{2}=0$.

\section{Stieltjes-type transforms for the coefficients and remainder terms}

Let us define

$$
v_{1}(z)=e^{-\lambda_{1} z} z^{-\mu_{1}-1} w_{1}(z), \quad v_{2}(z)=\mathrm{e}^{-\lambda_{1} z} z^{-\mu_{1}-1} w_{2}(z),
$$

so that for large $|z|$

$$
\begin{aligned}
& v_{1}(z) \sim \frac{1}{z} \sum_{s=0}^{\infty} \frac{a_{s, 1}}{z^{s}}, \quad|\operatorname{ph} z| \leqslant \frac{3}{2} \pi-\delta \\
& v_{2}(z) \sim \mathrm{e}^{z} z^{\mu_{2}-\mu_{1}-1} \sum_{s=0}^{\infty} \frac{a_{s_{2}}}{z^{s}}, \quad-\frac{1}{2} \pi+\delta \leqslant \operatorname{ph} z \leqslant \frac{5}{2} \pi-\delta
\end{aligned}
$$

compare (2.1), (2.2) and (2.3).

Lemma 3.1. Let $\rho$ be any constant such that $\rho-\delta$ exceeds the radius of the boundary of $\boldsymbol{A}, \dagger$ and $\mathcal{X}$ any constant in the closed interval $\left[-\frac{1}{2} \pi+\delta, \frac{1}{2} \pi-\delta\right]$. Then

$$
v_{1}(z)=-\frac{C_{1}}{2 \pi i} \int_{\rho \mathrm{e}}^{\infty(x+\pi) i} \frac{\infty \mathrm{e}^{(x+\pi) i}}{t-z} \mathrm{v} t-\frac{1}{2 \pi i} \int_{\rho \mathrm{e}}^{\rho \mathrm{e}^{(x-\pi) i}} \frac{v_{1}(t)}{t-z} \mathrm{~d} t
$$

valid when $z$ lies in the annular sector

$$
|z|>\rho, \quad \mathcal{X}-\pi<\operatorname{ph} z<\mathcal{X}+\pi .
$$

In (3.4) the path in the first integral is a straight line, and in the second integral it is an arc of the circle $|t|=\rho$ described in the positive sense.

Proof. From (2.7) and (3.1) we have

$$
C_{1} v_{2}(t)=v_{1}(t)-v_{1}\left(t e^{-2 \pi i}\right)
$$

Accordingly, if $r$ is any positive number exceeding $\rho$ and $|z|$, it follows that

$$
\begin{aligned}
& C_{1} \int_{\rho \mathrm{e}}^{r \mathrm{e}^{(x+\pi) i}} \frac{v_{2}(t)}{t-z} \mathrm{~d} t=\int_{\rho \mathrm{e}^{(x+\pi) i}}^{r \mathrm{e}^{(x+\pi) i}} \frac{v_{1}(t)}{t-z} \mathrm{~d} t-\int_{\rho \mathrm{e}}^{\mathrm{e}^{(x+\pi) i}} \frac{v_{1}\left(t \mathrm{e}^{-2 \pi i}\right)}{t-z} \mathrm{~d} t \\
& =\int_{\rho \mathrm{e}^{(x+\pi) i}}^{r \mathrm{e}^{(x+\pi) i}} \frac{v_{1}(t)}{t-z} \mathrm{~d} t-\int_{\rho \mathrm{e}^{(x-\pi) i}}^{r \mathrm{e}^{(x-x) i}} \frac{v_{1}(t)}{t-z} \mathrm{~d} t .
\end{aligned}
$$

By application of Cauchy's theorem to the contour indicated in figure 1, we obtain

$$
v_{1}(z)=-\frac{1}{2 \pi i}\left(\int_{\rho \mathrm{e}^{(x+\pi) i}}^{r \mathrm{e}^{(x+\pi) i}}+\int_{r \mathrm{e}^{(x+\pi) i}}^{r \mathrm{e}^{(x-\pi) i}}+\int_{r \mathrm{e}^{(x-\pi) i}}^{\rho \mathrm{e}^{(x-\pi) i}}+\int_{\rho \mathrm{e}^{(x-\pi) i}}^{\rho \mathrm{e}^{(x+\pi) i}}\right) \frac{v_{1}(t)}{t-z} \mathrm{~d} t .
$$

Let $r \rightarrow \infty$. Since $\mathcal{X}-\pi \geqslant-\frac{3}{2} \pi+\delta$ and $\mathcal{X}+\pi \leqslant \frac{3}{2} \pi-\delta$, it follows from (3.2) that $v_{1}(t)=\mathcal{O}(1 / r)$, uniformly on the circular arc of radius $r$. Hence the limiting

$\dagger$ Actually, until $\S 4$ it would suffice for $\rho$ to exceed the radius of the boundary of $A$.

Proc. R. Soc. Lond. A (1994) 


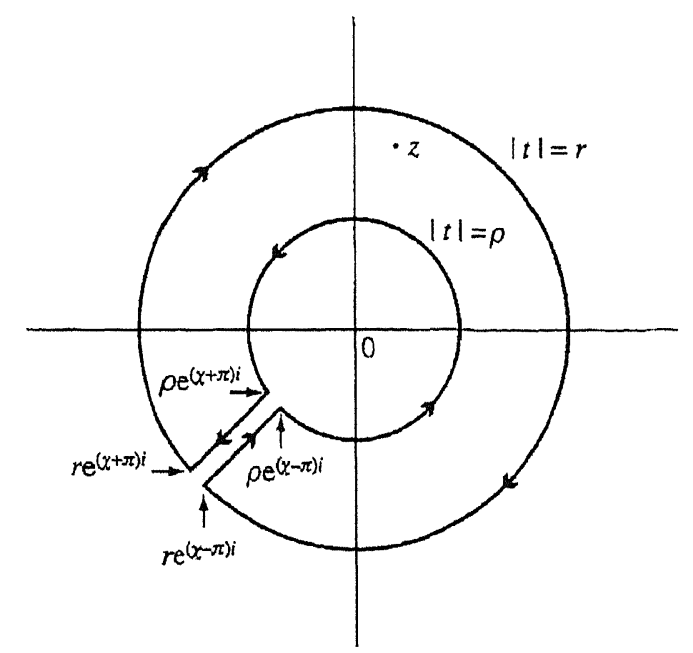

Figure 1. $t$-plane. Contour for equation (3.7).

value of the integral around this arc is zero. The desired result (3.4) is obtained by combining (3.6) and (3.7), and then taking the limit.

Lemma 3.2. Assume the conditions of Lemma 3.1, and let $n$ be an arbitrary non-negative integer. Then

$$
a_{s, 1}=\frac{1}{2 \pi i}\left\{(-)^{s-1} C_{1} \int_{\rho \mathrm{e}^{x_{i}}}^{\infty \mathrm{e}^{x_{i}}} v_{2}\left(t \mathrm{e}^{\pi i}\right) t^{s} \mathrm{~d} t+\int_{\rho \mathrm{e}}^{\rho(x-\pi) i} v_{1}(t) t^{s} \mathrm{~d} t\right\}
$$

and

$$
R_{n}^{(1)}(z)=-\frac{\mathrm{e}^{\lambda_{1} z} z^{\mu_{1}}}{2 \pi i z^{n-1}}\left\{(-)^{n} C_{1} \int_{\rho \mathrm{e}^{x_{i}}}^{\infty \mathrm{e}^{x_{i}}} \frac{v_{2}\left(t \mathrm{e}^{\pi i}\right) t^{n}}{t+z} \mathrm{~d} t+\int_{\rho \mathrm{e}^{(x-\pi) i}}^{\rho \mathrm{e}^{(\mathcal{x}+\pi) i}} \frac{v_{1}(t) t^{n}}{t-z} \mathrm{~d} t\right\} .
$$

Proof. To prove this result, we substitute into (3.4) by means of the expansion

$$
\frac{1}{t-z}=-\sum_{s=0}^{n-1} \frac{t^{s}}{z^{s+1}}+\frac{t^{n}}{z^{n}(t-z)}
$$

and subsequently replace $t$ by $t \mathrm{e}^{\pi i}$ in the integrals along the straight-line paths. We obtain

where

$$
w_{1}(z)=\mathrm{e}^{\lambda_{1} z} z^{\mu_{1}} \sum_{s=0}^{n-1} \frac{\tilde{a}_{s, 1}}{z^{s}}+\tilde{R}_{n}^{(1)}(z)
$$

$$
\tilde{a}_{s, 1}=\frac{1}{2 \pi i}\left\{(-)^{s-1} C_{1} \int_{\rho e^{x i}}^{\infty \mathrm{e}^{x_{i}}} v_{2}\left(t \mathrm{e}^{\pi i}\right) t^{s} \mathrm{~d} t+\int_{\rho \mathrm{e}^{(x-\pi) i}}^{\rho \mathrm{e}^{(x+\pi) i}} v_{1}(t) t^{s} \mathrm{~d} t\right\}
$$

and

$$
\tilde{R}_{n}^{(1)}(z)=-\frac{\mathrm{e}^{\lambda_{1} z} z^{\mu_{1}}}{2 \pi i z^{n-1}}\left\{(-)^{n} C_{1} \int_{\rho \mathrm{e}^{x_{i}}}^{\infty \mathrm{e}^{x_{i}}} \frac{v_{2}\left(t \mathrm{e}^{\pi i}\right) t^{n}}{t+z} \mathrm{~d} t+\int_{\rho \mathrm{e}^{(x-\pi) i}}^{\rho \mathrm{e}^{(x+\pi) i}} \frac{v_{1}(t) t^{n}}{t-z} \mathrm{~d} t\right\}
$$

Proc. R. Soc. Lond. A (1994) 
Obviously, Lemma 3.2 will follow if we can show that $\tilde{a}_{s, 1}=a_{s, 1}$ and $\tilde{R}_{n}^{(1)}(z)=$ $R_{n}^{(1)}(z)$. For this purpose we assume temporarily that $n$ is fixed and $z$ is large with $\mathrm{ph} z=0$. With the aid of (3.3) we easily deduce from (3.12) that in these circumstances we have

$$
\tilde{R}_{n}^{(1)}(z)=\mathrm{e}^{\lambda_{1} z} z^{\mu_{1}} \mathcal{O}\left(z^{-n}\right) .
$$

On comparing (3.10) and (3.13) with (2.2) and recalling that asymptotic expansions of Poincaré type are unique, we deduce that $\tilde{a}_{s, 1}=a_{s, 1}, s=0,1, \ldots, n-1$, and hence from (2.11) and (3.10) that $\tilde{R}_{n}^{(1)}(z)=R_{n}^{(1)}(z)$, (if ph $z=0$ or not).

\section{Proof of Theorem 2.1}

Lemma 4.1. Let $m$ be an arbitrary fixed non-negative integer, and $t$ lie in the annular sector

$$
|t| \geqslant \rho-\delta, \quad|\mathrm{ph} t| \leqslant \frac{3}{2} \pi-\delta
$$

Then

$$
v_{2}\left(t \mathrm{e}^{\pi i}\right)=-\mathrm{e}^{\left(\mu_{2}-\mu_{1}\right) \pi i} \mathrm{e}^{-t} t^{\mu_{2}-\mu_{1}-1}\left\{\sum_{j=0}^{m-1}(-)^{j} \frac{a_{j, 2}}{t^{j}}+\frac{\phi_{m}(t)}{t^{m}}\right\}
$$

where

$$
\left|\phi_{m}(t)\right| \leqslant \Phi_{m}
$$

and $\Phi_{m}$ is assignable independently of $t$.

This result is an immediate consequence of (3.3) and the fact that $v_{2}\left(t \mathrm{e}^{\pi i}\right)$ is analytic on the annular sector (4.1).

To prove Theorem 2.1 we set $\mathcal{X}=0$ in (3.8). We obtain

$$
a_{s, 1}=(-)^{s-1} \frac{C_{1}}{2 \pi i} \int_{\rho}^{\infty} v_{2}\left(t \mathrm{e}^{\pi i}\right) t^{s} \mathrm{~d} t+\frac{1}{2 \pi i} \int_{\rho e^{-\pi i}}^{\rho \mathrm{e}^{\pi i}} v_{1}(t) t^{s} \mathrm{~d} t
$$

In the second integral, $v_{1}(t)$ is bounded on the path of integration; accordingly

$$
\int_{\rho \mathrm{e}^{-\pi i}}^{\rho \mathrm{e}^{\pi i}} v_{1}(t) t^{s} \mathrm{~d} t=\mathcal{O}\left(\rho^{s}\right), \quad s \rightarrow \infty .
$$

In the first integral, we may substitute in the integrand by means of Lemma 4.1. This yields

$$
\begin{aligned}
\int_{\rho}^{\infty} v_{2}\left(t \mathrm{e}^{\pi i}\right) t^{s} \mathrm{~d} t=-\mathrm{e}^{\left(\mu_{2}-\mu_{1}\right) \pi i}\left\{\sum_{j=0}^{m-1}(-)^{j} a_{j, 2} \int_{\rho}^{\infty} \mathrm{e}^{-t} t^{s+\mu_{2}-\mu_{1}-j-1} \mathrm{~d} t\right. \\
\left.+\int_{\rho}^{\infty} \mathrm{e}^{-t} t^{s+\mu_{2}-\mu_{1}-m-1} \phi_{m}(t) \mathrm{d} t\right\}
\end{aligned}
$$

Now,

$$
\begin{aligned}
\int_{\rho}^{\infty} \mathrm{e}^{-t} t^{s+\mu_{2}-\mu_{1}-j-1} \mathrm{~d} t & =\Gamma\left(s+\mu_{2}-\mu_{1}-j\right)-\gamma\left(s+\mu_{2}-\mu_{1}-j, \rho\right) \\
& =\Gamma\left(s+\mu_{2}-\mu_{1}-j\right)+s^{-1} \mathcal{O}\left(\rho^{s}\right), \quad s \rightarrow \infty .
\end{aligned}
$$

Proc. R. Soc. Lond. A (1994) 
(This step follows from the well-known power-series expansion for the incomplete Gamma function, given for example in Olver $(1974, \mathrm{ch} .2, \S 5.1)$.) For the other integral in (4.5) we have

$$
\begin{aligned}
\left|\int_{\rho}^{\infty} \mathrm{e}^{-t} t^{s+\mu_{2}-\mu_{1}-m-1} \phi_{m}(t) \mathrm{d} t\right| & \leqslant \Phi_{m} \int_{\rho}^{\infty} \mathrm{e}^{-t} t^{s+\Re \mu_{2}-\Re \mu_{1}-m-1} \mathrm{~d} t \\
& <\Phi_{m} \Gamma\left(s+\Re \mu_{2}-\Re \mu_{1}-m\right),
\end{aligned}
$$

provided that $s>\Re \mu_{1}-\Re \mu_{2}+m$.

On combining the foregoing results we arrive at

$$
a_{s, 1}=(-)^{s} \frac{\mathrm{e}^{\left(\mu_{2}-\mu_{1}\right) \pi i}}{2 \pi i}\left\{C_{1} \sum_{j=0}^{m-1}(-)^{j} a_{j, 2} \Gamma\left(s+\mu_{2}-\mu_{1}-j\right)+\epsilon_{m, s}\right\}
$$

where, for large $s$,

$$
\epsilon_{m, s}=\mathcal{O}\left(\rho^{s}\right)+C_{1} \Gamma\left(s+\Re \mu_{2}-\Re \mu_{1}-m\right) \mathcal{O}(1) \subseteq \Gamma\left(s+\mu_{2}-\mu_{1}-m\right) \mathcal{O}(1) .
$$

This establishes (2.9). The proof of (2.10) is similar. $\dagger$

5. Proof of Theorem 2.2 for $w_{1}(z)$ when $|\operatorname{ph} z| \leqslant \pi-\delta$

From (3.9) with $\mathcal{X}=0$ we obtain

$$
R_{n}^{(1)}(z)=-\frac{e^{\lambda_{1} z} z^{\mu_{1}}}{2 \pi i z^{n-1}}\left\{(-)^{n} C_{1} \int_{\rho}^{\infty} \frac{v_{2}\left(t \mathrm{e}^{\pi i}\right) t^{n}}{t+z} \mathrm{~d} t+\int_{\rho \mathrm{e}^{-\pi i}}^{\rho \mathrm{e}^{\pi i}} \frac{v_{1}(t) t^{n}}{t-z} \mathrm{~d} t\right\}
$$

valid when $|z|>\rho$ and $|\mathrm{ph} z|<\pi$. For the second integral we note that $|t-z| \geqslant$ $|z|-\rho$ and that $\left|v_{1}(t)\right|$ is bounded on the integration path. In consequence, when $|z| \rightarrow \infty$ with ||$z|-n|$ bounded (compare (2.13)), we derive

$$
\int_{\rho \mathrm{e}^{-\pi i}}^{\rho \mathrm{e}^{\pi i}} \frac{v_{1}(t) t^{n}}{t-z} \mathrm{~d} t=\frac{1}{z} \mathcal{O}\left(\rho^{n}\right)
$$

uniformly for all values of $\mathrm{ph} z$.

In the first integral in (5.1) we may substitute by means of Lemma 4.1. From (1.7) we see that when $n>\Re\left(\mu_{1}-\mu_{2}\right)+m-1$

$$
\int_{0}^{\infty} \frac{\mathrm{e}^{-t} t^{n+\mu_{2}-\mu_{1}-j-1}}{t+z} \mathrm{~d} t=2 \pi \mathrm{e}^{z} z^{n+\mu_{2}-\mu_{1}-j-1} F_{n+\mu_{2}-\mu_{1}-j}(z), \quad|\mathrm{ph} z|<\pi
$$

Accordingly,

$$
\begin{array}{r}
\int_{\rho}^{\infty} \frac{v_{2}\left(t \mathrm{e}^{\pi i}\right) t^{n}}{t+z} \mathrm{~d} t=-\mathrm{e}^{\left(\mu_{2}-\mu_{1}\right) \pi i}\left\{2 \pi \mathrm{e}^{z} z^{n+\mu_{2}-\mu_{1}-1} \sum_{j=0}^{m-1}(-)^{j} a_{j, 2} \frac{F_{n+\mu_{2}-\mu_{1}-j}(z)}{z^{j}}\right. \\
\left.-S_{m, n}^{(1)}(z)+S_{m, n}^{(2)}(z)\right\}
\end{array}
$$

$\dagger$ Alternatively, we can interchange the roles of $w_{1}(z)$ and $w_{2}(z)$ as indicated in $\S 7$ below.

Proc. R. Soc. Lond. A (1994) 
where

$$
S_{m, n}^{(1)}(z)=\sum_{j=0}^{m-1}(-)^{j} a_{j, 2} \int_{0}^{\rho} \frac{e^{-t} t^{n+\mu_{2}-\mu_{1}-j-1}}{t+z} \mathrm{~d} t
$$

and

$$
S_{m, n}^{(2)}(z)=\int_{p}^{\infty} \frac{e^{-t} t^{n+\mu_{2}-\mu_{1}-m-1}}{t+z} \phi_{m}(t) \mathrm{d} t
$$

In $S_{m, n}^{(1)}(z)$ we have $|t+z| \geqslant|z|-\rho$. Hence

$$
\left|S_{m, n}^{(1)}(z)\right| \leqslant \frac{1}{|z|-\rho} \sum_{j=0}^{m-1}\left|a_{j, 2}\right| \gamma\left(n+\Re \mu_{2}-\Re \mu_{1}-j, \rho\right)=\frac{1}{|z| n} \mathcal{O}\left(\rho^{n}\right)
$$

as $|z| \rightarrow \infty$ with ||$z|-n|$ bounded, uniformly for all values of phz; compare (4.6).

For $S_{m, n}^{(2)}(z)$ we restrict $|p h z| \leqslant \pi-\delta(<\pi)$. Then $|t+z| \geqslant|z| \sin \delta$ on the integration path. Using also the bound (4.3), we find that when $n>\Re\left(\mu_{1}-\mu_{2}\right)+m$

$$
\left|S_{m, n}^{(2)}(z)\right| \leqslant \frac{\Phi_{m}}{|z| \sin \delta} \int_{\rho}^{\infty} \mathrm{e}^{-t} t^{n+\Re \mu_{2}-\Re \mu_{1}-m-1} \mathrm{~d} t<\frac{\Phi_{m} \Gamma\left(n+\Re \mu_{2}-\Re \mu_{1}-m\right)}{|z| \sin \delta} .
$$
that

On combining the foregoing results and referring to (2.1) and (2.14), we perceive

$$
R_{m, n}^{(1)}(z)=\mathrm{e}^{-z} z^{-n-\mu_{2}+\mu_{1}}\left\{\mathcal{O}\left(\rho^{n}\right)+\Gamma\left(n+\Re \mu_{2}-\Re \mu_{1}-m\right) \mathcal{O}(1)\right\} .
$$

Applying Stirling's formula and recalling again that $|z|$ and $n$ are related by (2.13), with $\alpha$ bounded, we see that when $|\mathrm{ph} z| \leqslant \pi-\delta$ we have

$$
R_{m, n}^{(1)}(z)=\mathrm{e}^{-z} z^{-n-\mu_{2}+\mu_{1}} \mathrm{e}^{-n} n^{n+\Re \mu_{2}-\Re \mu_{1}-m-\frac{1}{2}} \mathcal{O}(1) \subseteq \mathrm{e}^{-z-|z|} \mathcal{O}\left(z^{-m-\frac{1}{2}}\right) .
$$

This result agrees with (2.16); indeed, in the present circumstances it is stronger by a factor $\mathcal{O}\left(z^{-\frac{1}{2}}\right)$.

6. Proof of Theorem 2.2 for $w_{1}(z)$ when $|\mathrm{ph} z| \leqslant \frac{3}{2} \pi-3 \delta$

Lemma 6.1. Let $A_{1}$ and $\boldsymbol{A}_{2}$ denote the closed annular sectors

$$
\begin{aligned}
& \boldsymbol{A}_{1}=\left\{\tau:|\tau| \geqslant \rho, \quad-\frac{3}{2} \pi+2 \delta \leqslant \operatorname{ph} \tau \leqslant \frac{1}{2} \pi-2 \delta\right\}, \\
& \boldsymbol{A}_{2}=\left\{\tau:|\tau| \geqslant \rho+\delta, \quad-\frac{3}{2} \pi+3 \delta \leqslant \operatorname{ph} \tau \leqslant \frac{1}{2} \pi-3 \delta\right\} .
\end{aligned}
$$

Then

$$
\left|\frac{\phi_{m}(t)-\phi_{m}\left(z e^{-\pi i}\right)}{t+z}\right| \leqslant \Psi_{m}
$$

where $\phi_{m}(t)$ is defined as in Lemma 4.1 and $\Psi_{m}$ is assignable independently of $t$ and $z$, provided that $t \in \boldsymbol{A}_{1}$ and $z \mathrm{e}^{-\pi i} \in \boldsymbol{A}_{2}$.

Proof. This result can be proved in various ways. We shall proceed as follows. Write $\zeta=z \mathrm{e}^{-\pi i}$, so that

$$
\frac{\phi_{m}(t)-\phi_{m}\left(z \mathrm{e}^{-\pi i}\right)}{t+z}=\frac{\phi_{m}(t)-\phi_{m}(\zeta)}{t-\zeta}
$$

Proc. R. Soc. Lond. A (1994) 


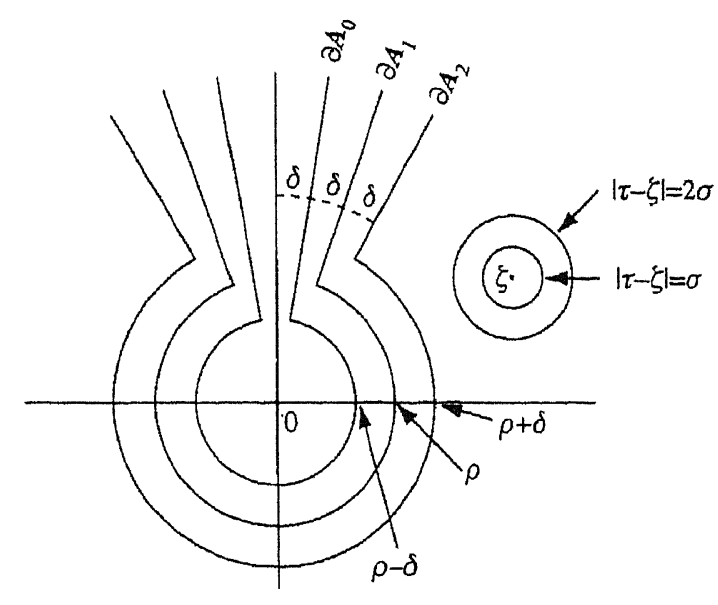

Figure 2. $\tau$-plane. $\partial A_{0}$ is the boundary of $A_{0}$, etc.

Also define

$$
A_{0}=\left\{\tau:|\tau| \geqslant \rho-\delta,-\frac{3}{2} \pi+\delta \leqslant \operatorname{ph} \tau \leqslant \frac{1}{2} \pi-\delta\right\} .
$$

Because $\rho-\delta$ exceeds the radius of the boundary of $\boldsymbol{A}$ (Lemma 3.1), we have $A \supset A_{0} \supset A_{1} \supset A_{2}$.

From figure 2 it is clear that we can find a positive constant $\sigma$ such that if $\zeta \in \boldsymbol{A}_{2}$, then the closed disc $|\tau-\zeta| \leqslant \sigma$ lies in $\boldsymbol{A}_{1}$ and the closed disc $|\tau-\zeta| \leqslant 2 \sigma$ lies in $\boldsymbol{A}_{0} . \dagger$ Accordingly, if $\zeta \in \boldsymbol{A}_{2}$ and $0<|t-\zeta| \leqslant \sigma$, then

$$
\frac{\phi_{m}(t)-\phi_{m}(\zeta)}{t-\zeta}=\frac{1}{2 \pi i} \int_{|\tau-\zeta|=2 \sigma} \frac{\phi_{m}(\tau)}{(\tau-t)(\tau-\zeta)} d \tau
$$

With the aid of Lemma 4.1 we then derive

$$
\left|\frac{\phi_{m}(t)-\phi_{m}(\zeta)}{t-\zeta}\right| \leqslant 2 \sigma \frac{\Phi_{m}}{\sigma \cdot 2 \sigma}=\frac{\Phi_{m}}{\sigma} .
$$

On the other hand, if $\zeta \in A_{2}$ and $t \in A_{1}$ with $|t-\zeta|>\sigma$, then we have directly

$$
\left|\frac{\phi_{m}(t)-\phi_{m}(\zeta)}{t-\zeta}\right| \leqslant \frac{2 \Phi_{m}}{\sigma}
$$

Combination of $(6.2),(6.3)$ and (6.4) establishes (6.1) (with $\Psi_{m}=2 \Phi_{m} / \sigma$ ), provided that $t \neq z \mathrm{e}^{-\pi i}$. The last condition is removed on replacing the lefthand side of ( 6.1$)$ by its limiting value.

$\dagger$ In fact, it suffices that $\sigma$ be bounded by $\delta$ and $\left(\frac{1}{2} \rho+\frac{1}{2} \delta\right) \sin 2 \delta$.

Proc. R. Soc. Lond. A (1994) 


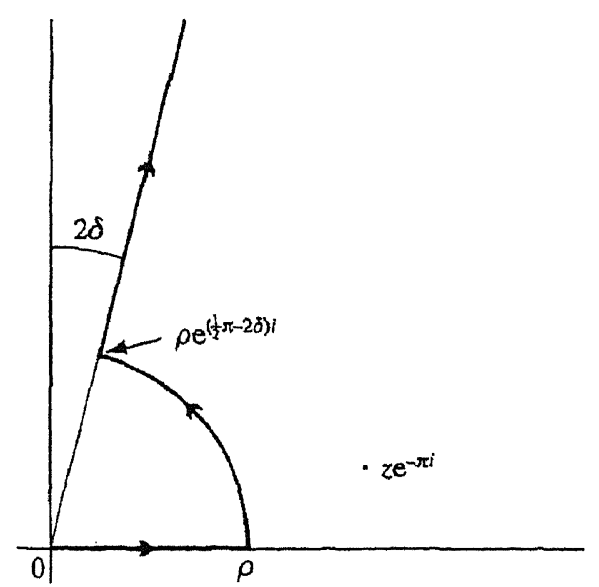

Figure 3. $t$-plane.

To continue the proof of Theorem 2.2 we set $\mathcal{X}=\frac{1}{2} \pi-2 \delta$ in (3.9). We obtain

$$
R_{n}^{(1)}(z)=-\frac{e^{\lambda_{1} z} z^{\mu_{1}}}{2 \pi i z^{n-1}}\left\{(-)^{n} C_{1} \int_{\rho e^{\left(\frac{1}{2} \pi-2 \delta\right) i}}^{\infty e^{\left(\frac{1}{2} \pi-2 \delta\right) i}} \frac{v_{2}\left(t \mathrm{e}^{\pi i}\right) t^{n}}{t+z} \mathrm{~d} t+\int_{\rho \mathrm{e}^{\left(-\frac{1}{2} \pi-2 \delta\right) i}}^{\rho \mathrm{e}^{\left(\frac{3}{2} \pi-26\right) i}} \frac{v_{1}(t) t^{n}}{t-z} \mathrm{~d} t\right\},
$$

valid when $|z|>\rho$ and $-\frac{1}{2} \pi-2 \delta<\operatorname{ph} z<\frac{3}{2} \pi-2 \delta$. As in (5.2) it follows that

$$
\int_{\rho e^{\left(-\frac{1}{2} \pi-2 \delta\right) i}}^{\rho e^{\left(\frac{3}{2} \pi-2 \delta\right) i}} \frac{v_{1}(t) t^{n}}{t-z} \mathrm{~d} t=\frac{1}{z} \mathcal{O}\left(\rho^{n}\right)
$$

as $|z| \rightarrow \infty$ with ||$z|-n|$ bounded.

Next, by substitution of (4.2) we obtain

$$
\begin{aligned}
\int_{\rho \mathrm{e}^{\left(\frac{1}{2} \pi-2 \delta\right) i}}^{\infty} \frac{v_{2}^{\left(\frac{1}{2} \pi-2 \delta\right) i}\left(t \mathrm{e}^{\pi i}\right) t^{n}}{t+z} \mathrm{~d} t & \\
=-\mathrm{e}^{\left(\mu_{2}-\mu_{1}\right) \pi i} & \left\{\sum_{j=0}^{m-1}(-)^{j} a_{j, 2} \int_{\rho e^{\left(\frac{1}{2} \pi-2 \delta\right) i}}^{\infty \mathrm{e}^{\left(\frac{1}{2} \pi-2 \delta\right) i}} \frac{\mathrm{e}^{-t} t^{n+\mu_{2}-\mu_{1}-j-1}}{t+z} \mathrm{~d} t\right. \\
& \left.+\int_{\rho \mathrm{e}^{\left(\frac{1}{2} \pi-2 \delta\right) i}}^{\infty e^{\left(\frac{1}{2} \pi-2 \delta\right) i}} \frac{\mathrm{e}^{-t} t^{n+\mu_{2}-\mu_{1}-m-1}}{t+z} \phi_{m}(t) \mathrm{d} t\right\} .
\end{aligned}
$$

Bearing in mind that $\left|z \mathrm{e}^{-\pi i}\right|>\rho$ and $-\frac{3}{2} \pi-2 \delta<\operatorname{ph}\left(z \mathrm{e}^{-\pi i}\right)<\frac{1}{2} \pi-2 \delta$ we may deform the path for the integrals under the summation sign, as indicated in figure 3 . Thus we have

$$
\begin{aligned}
\int_{\rho \mathrm{e}^{\left(\frac{1}{2} \pi-2 \delta\right) i}}^{\infty} \frac{\mathrm{e}^{\left(\frac{1}{2} \pi-2 \delta\right) t} t^{n+\mu_{2}-\mu_{1}-j-1}}{t+z} \mathrm{~d} t \\
=\left(\int_{0}^{\infty e^{\left(\frac{1}{2} \pi-2 \delta\right) i}}-\int_{\rho}^{\rho e^{\left(\frac{1}{2} \pi-2 \delta\right) i}}-\int_{0}^{\rho}\right) \frac{\mathrm{e}^{-t} t^{n+\mu_{2}-\mu_{1}-j-1}}{t+z} \mathrm{~d} t . \quad(6.8)
\end{aligned}
$$

Proc. R. Soc. Lond. A (1994) 
By analytic continuation of (5.3) it follows that

$$
\int_{0}^{\infty e^{\left(\frac{3}{2} \pi-2 \delta\right) i}} \frac{\mathrm{e}^{-t} t^{n+\mu_{2}-\mu_{1}-j-1}}{t+z} \mathrm{~d} t=2 \pi \mathrm{e}^{z} z^{n+\mu_{2}-\mu_{1}-j-1} F_{n+\mu_{2}-\mu_{1}-j}(z) .
$$

And as in the derivation of (5.2) and (5.5) we see that the contributions of the second and third integrals in the right member of $(6.8)$ are $z^{-1} \mathcal{O}\left(\rho^{n}\right)$ and $(z n)^{-1} \mathcal{O}\left(\rho^{n}\right)$ respectively. Hence we have

$$
\begin{aligned}
& \sum_{j=0}^{m-1}(-)^{j} a_{j, 2} \int_{\rho e^{\left(\frac{1}{2} \pi-2 \delta\right) i}}^{\infty e^{\left(\frac{1}{2} \pi-2 \delta\right) i}} \frac{\mathrm{e}^{-t} t^{n+\mu_{2}-\mu_{1}-j-1}}{t+z} \mathrm{~d} t \\
& =2 \pi \mathrm{e}^{z} z^{n+\mu_{2}-\mu_{1}-1} \sum_{j=0}^{m-1}(-)^{j} a_{j, 2} \frac{F_{n+\mu_{2}-\mu_{1}-j}(z)}{z^{j}}+\frac{1}{z} \mathcal{O}\left(\rho^{n}\right) .
\end{aligned}
$$

To estimate the remaining integral in (6.7) we decompose it as follows:

$$
\int_{\rho \mathrm{e}^{\left(\frac{1}{2} \pi-2 \delta\right) i}}^{\infty \mathrm{e}^{\left(\frac{1}{2} \pi-2 \delta\right) i}} \frac{\mathrm{e}^{-t} t^{n+\mu_{2}-\mu_{1}-m-1}}{t+z} \phi_{m}(t) \mathrm{d} t=T_{m, n}^{(1)}(z)+T_{m, n}^{(2)}(z)
$$

where

$$
\begin{aligned}
& T_{m, n}^{(1)}(z)=\phi_{m}\left(z \mathrm{e}^{-\pi i}\right) \int_{\rho \mathrm{e}^{\left(\frac{1}{2} \pi-2 \delta\right) i}}^{\infty \mathrm{e}^{\left(\frac{1}{2} \pi-2 \delta\right) t}} \frac{\mathrm{e}^{-t} t^{n+\mu_{2}-\mu_{1}-m-1}}{t+z} \mathrm{~d} t \\
& T_{m, n}^{(2)}(z)=\int_{\rho \mathrm{e}^{\left(\frac{1}{2} \pi-2 \delta\right) i}}^{\infty \mathrm{e}^{\left(\frac{1}{2} \pi-2 \delta\right) i}} \mathrm{e}^{-t} t^{n+\mu_{2}-\mu_{1}-m-1} \frac{\phi_{m}(t)-\phi_{m}\left(z \mathrm{e}^{-\pi i}\right)}{t+z} \mathrm{~d} t
\end{aligned}
$$

and until the final paragraph of this section we restrict

$$
|z| \geqslant \rho+\delta, \quad-\frac{1}{2} \pi+3 \delta \leqslant \operatorname{ph} z \leqslant \frac{3}{2} \pi-3 \delta,
$$

or, equivalently, $z \mathrm{e}^{-\pi i} \in \boldsymbol{A}_{2}$.

From Lemma 4.1 we know that $\left|\phi_{m}\left(z \mathrm{e}^{-\pi i}\right)\right| \leqslant \Phi_{m}$ when conditions (6.13) are satisfied. Hence we have

$$
T_{m, n}^{(1)}(z)=\mathrm{e}^{z} z^{n+\mu_{2}-\mu_{1}-m-1} F_{n+\mu_{2}-\mu_{1}-m}(z) \mathcal{O}(1)+\frac{1}{z} \mathcal{O}\left(\rho^{n}\right) ;
$$

compare (6.8) and (6.9).

For $T_{m, n}^{(2)}(z)$ we observe that the integrand in $(6.12)$ is analytic at $t=z \mathrm{e}^{-\pi i}$. Therefore we may deform the integration path in the following way:

$$
\int_{\rho \mathrm{e}^{\left(\frac{1}{2} \pi-26\right) t}}^{\infty \mathrm{e}^{\left(\frac{1}{2} \pi-2 \delta\right) i}}=\int_{\rho}^{\infty}-\int_{\rho}^{\rho \mathrm{e}^{\left(\frac{1}{2} \pi-26\right) i}} ;
$$

again see figure 3. By application of Lemma 6.1 we have

$$
\begin{array}{rl}
\mid \int_{\rho}^{\infty} \mathrm{e}^{-t} t^{n+\mu_{2}-\mu_{1}-m-1} \frac{\phi_{m}(t)-\phi_{m}\left(z \mathrm{e}^{-\pi i}\right)}{t}+z & \mathrm{~d} t \mid \\
& \leqslant \Psi_{m} \int_{\rho}^{\infty} \mathrm{e}^{-t} t^{n+\Re \mu_{2}-\Re \mu_{1}-m-1} \mathrm{~d} t \\
& <\Psi_{m} \Gamma\left(n+\Re \mu_{2}-\Re \mu_{1}-m\right),
\end{array}
$$

Proc. R. Soc. Lond. A (1994) 
and

$$
\begin{aligned}
& \left|\int_{\rho}^{\rho \mathrm{e}^{\left(\frac{1}{2} \pi-2 \delta\right) i}} \mathrm{e}^{-t} t^{n+\mu_{2}-\mu_{1}-m-1} \frac{\phi_{m}(t)-\phi_{m}\left(z \mathrm{e}^{-\pi i}\right)}{t+z} \mathrm{~d} t\right| \\
& \leqslant \Psi_{m} \int_{\rho}^{\rho \mathrm{e}^{\left(\frac{1}{2} \pi-2 \delta\right) i}}\left|\mathrm{e}^{-t} t^{n+\mu_{2}-\mu_{1}-m-1} \mathrm{~d} t\right|=\mathcal{O}\left(\rho^{n}\right) .
\end{aligned}
$$

On combining the foregoing results we obtain

$$
T_{m, n}^{(2)}(z)=\Gamma\left(n+\mu_{2}-\mu_{1}-m\right) \mathcal{O}(1)+\mathcal{O}\left(\rho^{n}\right) .
$$

If we substitute into (6.10) by means of (6.14) and (6.15) and then combine the $\mathcal{O}$-terms, we derive

$$
\begin{aligned}
& \int_{\rho e^{\left(\frac{1}{2} \pi-2 \delta\right) i}}^{\infty e^{\left(\frac{3}{2} \pi-2 \delta\right) i}} \frac{\mathrm{e}^{-t} t^{n+\mu_{2}-\mu_{1}-m-1}}{t+z} \phi_{m}(t) \mathrm{d} t=\mathrm{e}^{z} z^{n+\mu_{2}-\mu_{1}-m-1} F_{n+\mu_{2}-\mu_{1}-m}(z) \mathcal{O}(1) \\
& +\Gamma\left(n+\mu_{2}-\mu_{1}-m\right) \mathcal{O}(1) \text {. }
\end{aligned}
$$

The results (6.9) and (6.16) enable us to evaluate the right-hand side of (6.7). Thus we have

$$
\begin{aligned}
\int_{\rho \mathrm{e}^{\left(\frac{1}{2} \pi-2 \delta\right) i}}^{\infty e^{\left(\frac{1}{2} \pi-2 \delta\right) i}} \frac{v_{2}\left(t \mathrm{e}^{\pi i}\right) t^{n}}{t+z} \mathrm{~d} t= & -2 \pi \mathrm{e}^{\left(\mu_{2}-\mu_{1}\right) \pi i} \mathrm{e}^{z} z^{n+\mu_{2}-\mu_{1}-1} \\
& \times\left\{\sum_{j=0}^{m-1}(-)^{j} a_{j, 2} \frac{F_{n+\mu_{2}-\mu_{1}-j}(z)}{z^{j}}+\frac{F_{n+\mu_{2}-\mu_{1}-m}(z)}{z^{m}} \mathcal{O}(1)\right\} \\
& +\Gamma\left(n+\mu_{2}-\mu_{1}-m\right) \mathcal{O}(1) .
\end{aligned}
$$

We now return to (6.5). On substituting by means of (6.6) and (6.17) we see that equation (2.14) applies with the estimate

$$
\begin{aligned}
& R_{m, n}^{(1)}(z)=C_{1} z^{-m} F_{n+\mu_{2}-\mu_{1}-m}(z) \mathcal{O}(1) \\
& \quad+C_{1} \mathrm{e}^{-z} z^{-n-\mu_{2}+\mu_{1}+1} \Gamma\left(n+\mu_{2}-\mu_{1}-m\right) \mathcal{O}(1)+\mathrm{e}^{-z} z^{-n-\mu_{2}+\mu_{1}} \mathcal{O}\left(\rho^{n}\right) .
\end{aligned}
$$

From equations (2.9) and (2.11) of Olver (1993), we have

$$
z^{-m} F_{n+\mu_{2}-\mu_{1}-m}(z)= \begin{cases}\mathcal{O}\left(e^{-z-|z|} z^{-m}\right), & -\frac{1}{2} \pi+3 \delta \leqslant \mathrm{ph} z \leqslant \pi \\ \mathcal{O}\left(z^{-m}\right), & \pi \leqslant \mathrm{ph} z \leqslant \frac{3}{2} \pi-3 \delta\end{cases}
$$

Furthermore, from Stirling's formula we deduce that

$$
\mathrm{e}^{-z} z^{-n-\mu_{2}+\mu_{1}+1} \Gamma\left(n+\mu_{2}-\mu_{1}-m\right)=\mathcal{O}\left\{\mathrm{e}^{-z-|z|} z^{\frac{1}{2}-m}\right\}
$$

compare (5.6). And the remaining term in (6.18) is absorbable in these estimates. Accordingly, we arrive at

$$
R_{m, n}^{(1)}(z)= \begin{cases}\mathcal{O}\left(\mathrm{e}^{-z-|z|} z^{\frac{1}{2}-m}\right), & -\frac{1}{2} \pi+3 \delta \leqslant \mathrm{ph} z \leqslant \pi \\ \mathcal{O}\left(z^{\frac{1}{2} \sim m}\right), & \pi \leqslant \mathrm{ph} z \leqslant \frac{3}{2} \pi-3 \delta\end{cases}
$$

These results agree with (2.16) and (2.17) in the corresponding phase ranges, except that $z^{-m}$ is replaced by $z^{\frac{1}{2}-m}$. To strengthen the estimates into the required Proc. R. Soc. Lond. A (1994) 
form we re-expand the remainder term in the usual manner; thus

$$
R_{m, n}^{(1)}(z)=(-)^{m} C_{1} a_{m, 2} \frac{F_{n+\mu_{2}-\mu_{1}-m}(z)}{z^{m}}+R_{m+1, n}^{(1)}(z) .
$$

This completes the proof of Theorem 2.2 for the solution $w_{1}(z)$ in the phase range $-\frac{1}{2} \pi+3 \delta \leqslant \mathrm{ph} z \leqslant \frac{3}{2} \pi-3 \delta$. The corresponding extension to the range $-\frac{3}{2} \pi+3 \delta \leqslant \mathrm{ph} z \leqslant \frac{1}{2} \pi-3 \delta$ may be carried out in an analogous manner: for example, the integration paths everywhere are replaced by their conjugates, and $\phi_{m}\left(z \mathrm{e}^{-\pi i}\right)$ is replaced by $\phi_{m}\left(z \mathrm{e}^{\pi i}\right)$. Alternatively, we can appeal to symmetry.

\section{Completion of the proof of Theorem 2.2}

On replacing $3 \delta$ by $\delta$ in the results of $\S 6$, we see that equations (2.11), (2.14), (2.16) and (2.17) are established, except that the region of validity in (2.17) is restricted to $\pi \leqslant|\mathrm{ph} z| \leqslant \frac{3}{2} \pi-\delta$.

The corresponding results for the solution $w_{2}(z)$ can be arrived at by replacing the independent variable in the original equations (1.1) and (1.2) with $\zeta=z \mathrm{e}^{-\pi i}$. Thus we have

where

$$
\frac{d^{2} w}{d \zeta^{2}}+f^{*}(\zeta) \frac{d w}{d \zeta}+g^{*}(\zeta) w=0
$$

Hence

$$
f^{*}(\zeta)=-f(-\zeta), \quad g^{*}(\zeta)=g(-\zeta)
$$

$$
f^{*}(\zeta)=\sum_{s=0}^{\infty}(-)^{s-1} \frac{f_{s}}{\zeta^{s}}, \quad g^{*}(\zeta)=\sum_{s=0}^{\infty}(-)^{s} \frac{g_{s}}{\zeta^{s}}, \quad \zeta \in \boldsymbol{A} ;
$$

compare (1.3). In the expansions that correspond to (2.2) and (2.3) the characteristic values are $\lambda_{1}^{*}$ and $\lambda_{2}^{*}$, where $\lambda_{1}^{*}=-\lambda_{2}, \lambda_{2}^{*}=-\lambda_{1}$. Accordingly, $\lambda_{2}^{*}-\lambda_{1}^{*}=1$; compare (2.1). Similarly, the exponents are found to be $\mu_{1}^{*}=\mu_{2}, \mu_{2}^{*}=\mu_{1}$. If we apply the part of Theorem 2.2 that we have established so far and then restore the variable $z=\zeta \mathrm{e}^{\pi i}$, we arrive at (2.12), (2.15), (2.18) and (2.19) except, again, the region of validity of (2.19) is restricted: in this case to the union of the sectors $-\frac{1}{2} \pi+\delta \leqslant \operatorname{ph} z \leqslant 0$ and $2 \pi \leqslant \operatorname{ph} z \leqslant \frac{5}{2} \pi-\delta$. The analysis is straightforward and there is no need to record the details.

The remaining task is to attain the full regions of validity of (2.17) and (2.19). As in the case of the confluent hypergeometric function (Olver 1991b, 1993) we shall achieve this by use of connection formulae.

First, we consider the solution $w_{1}(z)$. From (2.5) of Olver (1993), with $k=-1$ and $p=n+\mu_{2}-\mu_{1}-s$, we obtain

$$
F_{n+\mu_{2}-\mu_{1}-s}\left(z \mathrm{e}^{-2 \pi i}\right)=(-)^{n-s-1} i \mathrm{e}^{\left(\mu_{2}-\mu_{1}\right) \pi i}+\mathrm{e}^{2\left(\mu_{2}-\mu_{1}\right) \pi i} F_{n+\mu_{2}-\mu_{1}-s}(z) .
$$

Hence from (2.14) we have

$$
\begin{aligned}
& \mathrm{e}^{2 \mu_{1} \pi i} R_{n}^{(1)}\left(z \mathrm{e}^{-2 \pi i}\right)=(-)^{n-1} i \mathrm{e}^{\left(\mu_{2}-\mu_{1}\right) \pi i} C_{1} \mathrm{e}^{\lambda_{2} z} z^{\mu_{2}} \sum_{s=0}^{m-1}(-)^{s} a_{s, 2} \frac{F_{n+\mu_{2}-\mu_{1}-s}(z)}{z^{s}} \\
&-C_{1} \mathrm{e}^{\lambda_{2} z} z^{\mu_{2}} \sum_{s=0}^{m-1} \frac{a_{s, 2}}{z^{s}}-(-)^{n} i \mathrm{e}^{\left(\mu_{1}-\mu_{2}\right) \pi i} \mathrm{e}^{\lambda_{2} z} z^{\mu_{2}} R_{m, n}^{(1)}\left(z \mathrm{e}^{-2 \pi i}\right) .
\end{aligned}
$$

Proc. R. Soc. Lond. A. (1994) 
On substituting into (2.7) by means of (2.11) and (2.12) then referring to (2.14) and (7.2), we find that on reduction

$$
R_{m, n}^{(1)}(z)=\mathrm{e}^{2\left(\mu_{1}-\mu_{2}\right) \pi i} R_{m, n}^{(1)}\left(z \mathrm{e}^{-2 \pi i}\right)+\hat{R}_{m, n}^{(1)}(z)+\hat{R}_{n}^{(2)}(z)
$$

where

$$
\begin{aligned}
& \hat{R}_{m, n}^{(1)}(z)=(-)^{n} i \mathrm{e}^{\left(\mu_{1}-\mu_{2}\right) \pi i} C_{1} \sum_{s=m}^{n-1} \frac{a_{s, 2}}{z^{s}} \\
& \hat{R}_{n}^{(2)}(z)=(-)^{n} i \mathrm{e}^{\left(\mu_{1}-\mu_{2}\right) \pi i} C_{1} \mathrm{e}^{-\lambda_{2} z} z^{-\mu_{2}} R_{n}^{(2)}(z) .
\end{aligned}
$$

We now let $z \rightarrow \infty$ in the sector $\frac{3}{2} \pi-\delta \leqslant \operatorname{ph} z \leqslant \frac{5}{2} \pi-\delta$ with ||$z|-n|$ bounded.

From (2.16) we immediately derive

$$
\mathrm{e}^{2\left(\mu_{1}-\mu_{2}\right) \pi i} R_{m, n}^{(1)}\left(z \mathrm{e}^{-2 \pi i}\right)=\mathcal{O}\left(\mathrm{e}^{-|z|-z} z^{-m}\right) \subseteq \mathcal{O}\left(z^{-m}\right) .
$$

Next, consider $\hat{R}_{m, n}^{(1)}(z)$. If we write $\hat{\alpha}=\sup \alpha$, then we have $|z| \geqslant n-\hat{\alpha}$; see (2.13). From Theorem 2.1 we know that

$$
a_{s, 2}=\mathcal{O}\left\{\Gamma\left(s+\mu_{1}-\mu_{2}\right)\right\}, \quad s \rightarrow \infty .
$$

Let $M$ be the least positive integer that satisfies $M \geqslant m$ and $M \geqslant \hat{\alpha}$. Applying Stirling's formula, we see that there is an assignable constant $A$ such that

$$
\left|a_{s, 2}\right| \leqslant A \mathrm{e}^{-s} s^{s+\Re\left(\mu_{1}-\mu_{2}\right)-\frac{1}{2}}, \quad s \geqslant M .
$$

Therefore when $n \geqslant M$ we have

$$
\begin{aligned}
\left|\sum_{s=m}^{n-1} \frac{a_{s, 2}}{z^{s}}\right| & \leqslant \sum_{s=m}^{M-1} \frac{\left|a_{s, 2}\right|}{|z|^{s}}+\frac{A}{|z|^{M}} \sum_{s=M}^{n-1} \frac{e^{-s} s^{s+\Re\left(\mu_{1}-\mu_{2}\right)-\frac{1}{2}}}{(n-\hat{\alpha})^{s-M}} \\
& \leqslant \sum_{s=m}^{M-1} \frac{\left|a_{s, 2}\right|}{|z|^{s}}+\frac{A}{|z|^{M}} \sum_{s=M}^{\infty} \frac{e^{-s} s^{s+\Re\left(\mu_{1}-\mu_{2}\right)-\frac{1}{2}}}{(s+1-\hat{\alpha})^{s-M}} .
\end{aligned}
$$

The infinite sum converges; hence we see that

$$
\hat{R}_{m, n}^{(1)}(z)=\mathcal{O}\left(z^{-m}\right)
$$

Lastly, in the second paragraph of this section we showed that the region of validity of (2.18) includes the sector $\frac{3}{2} \pi-\delta \leqslant \mathrm{ph} z \leqslant 2 \pi$, and that of (2.19) includes $2 \pi \leqslant \mathrm{ph} z \leqslant \frac{5}{2} \pi-\delta$. Substituting into $(2.15)$ by means of these estimates and using also Olver (1993, eqns (2.9) and (2.11)) with $z$ replaced by $z \mathrm{e}^{-\pi i}$ and $p=n+\mu_{1}-\mu_{2}-s$, we arrive at

$$
\begin{array}{ll}
R_{n}^{(2)}(z)=\mathcal{O}\left(\mathrm{e}^{\lambda_{1} z} z^{\mu_{1}} \mathrm{e}^{z-|z|}\right), & \frac{3}{2} \pi-\delta \leqslant \mathrm{ph} z \leqslant 2 \pi, \\
R_{n}^{(2)}(z)=\mathcal{O}\left(\mathrm{e}^{\lambda_{1} z} z^{\mu_{1}}\right), & 2 \pi \leqslant \operatorname{ph} z \leqslant \frac{5}{2} \pi-\delta .
\end{array}
$$

Correspondingly,

$$
\begin{aligned}
& \hat{R}_{n}^{(2)}(z)=\mathcal{O}\left(\mathrm{e}^{-|z|} z^{\mu_{1}-\mu_{2}}\right), \quad \frac{3}{2} \pi-\delta \leqslant \mathrm{ph} z \leqslant 2 \pi \\
& \hat{R}_{n}^{(2)}(z)=\mathcal{O}\left(\mathrm{e}^{-z} z^{\mu_{1}-\mu_{2}}\right), \quad 2 \pi \leqslant \mathrm{ph} z \leqslant \frac{5}{2} \pi-\delta .
\end{aligned}
$$

Thus in both cases we may assert that

$$
\hat{R}_{n}^{(2)}(z)=\mathcal{O}\left(z^{-m}\right)
$$

Proc. R. Soc. Lond. A (1994) 
On substituting into (7.3) by means of (7.4), (7.5) and (7.6), we obtain the required result

$$
R_{m, n}^{(1)}(z)=\mathcal{O}\left(z^{-m}\right), \quad \frac{3}{2} \pi-\delta \leqslant \operatorname{ph} z \leqslant \frac{5}{2} \pi-\delta .
$$

The extension of the region of validity of $(2.17)$ to the sector $-\frac{5}{2} \pi+\delta \leqslant \mathrm{ph} z \leqslant$ $-\frac{3}{2} \pi+\delta$ may be carried out by similar analysis. And the extension of (2.19) to the sectors $-\frac{3}{2} \pi+\delta \leqslant \operatorname{ph} z \leqslant-\frac{1}{2} \pi+\delta$ and $\frac{5}{2} \pi-\delta \leqslant \operatorname{ph} z \leqslant \frac{7}{2} \pi-\delta$ is then achieved by the procedure outlined in the second paragraph of this section.

Another extension of the foregoing analysis shows that if $C_{1} C_{2} \neq 0$, then (7.7) breaks down when $\mathrm{ph} z$ crosses $\frac{5}{2} \pi$. This is because (7.4) and (7.5) continue to hold, but in the vicinity of $\mathrm{ph} z=\frac{5}{2} \pi$ we have

$$
R_{n}^{(2)}(z)=-\mathrm{e}^{2\left(\mu_{2}-\mu_{1}\right) \pi i} \mathrm{e}^{\lambda_{1} z} z^{\mu_{1}}\left\{C_{2}+\mathcal{O}\left(z^{-1}\right)\right\}
$$

compare again (2.15), (2.19) and Olver (1993, eqn (2.11)). Hence

$$
\hat{R}_{n}^{(2)}(z)=(-)^{n-1} i \mathrm{e}^{\left(\mu_{2}-\mu_{1}\right) \pi i} C_{1} \mathrm{e}^{-z} z^{\mu_{1}-\mu_{2}}\left\{C_{2}+\mathcal{O}\left(z^{-1}\right)\right\}
$$

and this is exponentially large when ph $z$ exceeds $\frac{5}{2} \pi$. A similar observation applies to the other boundary $\operatorname{ph} z=-\frac{5}{2} \pi+\delta$, and also to the boundaries $\operatorname{ph} z=-\frac{3}{2} \pi+\delta$ and $\mathrm{ph} z=\frac{7}{2} \pi-\delta$ in (2.19).

The proof of Theorem 2.2 is complete.

\section{Conclusions}

In this paper we have considered the asymptotic expansions of solutions of the general homogeneous linear differential equation of the second order in the neighbourhood of an irregular singularity of rank one. We have shown that if these expansions are truncated at (or near) their optimal stage, then the remainder term can be re-expanded as a series of $F$-functions (generalized exponential integrals), divided by rising powers of $-z, z$ being the independent variable. Furthermore, the coefficients are the same as those in the original asymptotic expansion of the complementary solution.

The total sector of validity of each of the new expansions has an angle of $5 \pi-2 \delta$, compared with $3 \pi-2 \delta$ for the original Poincaré forms, $\delta$ being an arbitrary small positive constant. Moreover, the new expansions are considerably more accurate and also provide a smooth interpretation of the Stokes phenomenon.

We have also shown how to construct explicit asymptotic expansions for the bigher coefficients in the original asymptotic solutions of the differential equation in terms of inverse factorials. Again, the coefficients are the lower coefficients of the complementary solution.

Our method of proof is to construct Stieltjes-type transforms for the remainder terms from the standard connection formulae for the solutions, and then to derive the required results by appropriate expansions of the integrands. Necessary extensions of the regions of validity are found by use, again, of the connection formulae. The method appears to be fairly general, and could be applied, for example, to linear differential equations of other orders or with other types of singularity.

We are indebted to the referees for several improvements in the presentation of the results. The

Proc. R. Soc. Lond. A (1994) 
research of F. W. J. O. has been supported by the U.S. National Science Foundation under grants DMS 87.23039 and DMS 92-08690.

\section{References}

Berry, M. V. 1950 Waves near Stokes lines. Proc. R. Soc. Lond. A427, 265-280.

Berry, M. V. 1991 Asymptotics, superasymptotics, hyperasymptotics, ... In Asymptotics beyond all orders (ul. H. Segur, S. Tanveer \& H. Levine), ser. B (Physics), vol. 284, pp. 1-14. New York: Plemm Press.

Berry, M. Y.\& Howls, C. J. 1991 Hyperasymptotics for integrals with saddles. Proc. R. Soc. Lond. A434, 657. (755.

Boyd, W. ('. (., 1990 Stielt.jes transforms and the Stokes phenomenon. Proc. R. Soc. Lond. A $429,227-2.16$.

McLeod, J. B. 1992 Snoothing of Stokes discontinuities. Proc. R. Soc. Lond. A437, 343-354.

Olver, F. W. J. 1974 Asymptotics and special functions. New York: Academic Press.

Olver, F. II. J. 1990 On Stokes' phenomenon and converging factors. In Asymptotic and computational onalysis (ed. R. Wong), pp. 329-355. New York: Marcel Dekker.

Olver, F. W. J. loyla Uniform, exponentially improved, asymptotic expansions for the generalized expencutial integral. SIAM J. math. Analysis 22, 1460-1474.

Olver, F. If. J. 19916 Uniform, exponentially improved, asymptotic expansions for the confluent hypergentric function and other integral transforms. SIAM J. math. Analysis 22, 14751489.

Olver, F. W. J. 19e?] Fixponentially-improved asymptotic solutions of ordinary differential equations I: The cunflurm hypersecometric function. SIAM J. math. Analysis 24, 756-767.

Olver, F. W. J. I59.1 Asymptotic expansions of the coefficients in asymptotic series solutions of linear differential requations. Methods appl. Analysis 1, 1-13.

Paris, R. B. 10gia Smustling of the Stokes phenomenon for high-order differential equations. Proc. R. Soc. Loml. A436, 165-180.

Paris, R. B. 1992b Smouthing of the Stokes phenomenon using Mellin-Barnes integrals. J. Comp. appl. Math. 41,117 133.

Recrivel 201 April 1993; accepted 7 September 1993

Proc. R. Soc. Lord. A (1)!H) 